\title{
New supersonic gas jet target for low energy nuclear reaction studies
}

\author{
F. Favela, ${ }^{1}$ L. Acosta ${ }^{1,2}$ E. Andrade, ${ }^{1}$ V. Araujo, ${ }^{1}$ A. Huerta, ${ }^{1}$ O. G. de Lucio, ${ }^{1}$ G. Murillo, ${ }^{3}$ \\ M. E. Ortiz, ${ }^{1}$ R. Policroniades, ${ }^{3}$ P. Santa Rita, ${ }^{1}$ A. Varela, ${ }^{4}$ and E. Chávez ${ }^{1}$ \\ ${ }^{1}$ Instituto de Física, Universidad Nacional Autónoma de México, 01000, México, D.F., Mexico \\ ${ }^{2}$ INFN-Sezione di Catania, Via Santa Sofía 64 I-95123, Catania, Italy \\ ${ }^{3}$ Departamento de Aceleradores, Instituto Nacional de Investigaciones Nucleares, \\ Carretera México-Toluca S/N, Ocoyoacac, Estado de México, 52750, Mexico \\ ${ }^{4}$ Centro de Ciencias de la Atmósfera, Universidad Nacional Autónoma de México, \\ 01000, México, D.F., Mexico
}

(Received 3 July 2015; published 29 December 2015)

\begin{abstract}
A windowless supersonic gas jet target (SUGAR) has been put in operation recently in Mexico. It is the first target of its kind in the country and the region. New research opportunities become available with this facility through the study of the direct beam-gas interaction: nuclear physics and astrophysics, atomic physics, interaction of radiation with matter and other interdisciplinary applications. A general description of the apparatus and its commissioning is given here. Air, nitrogen and argon jets were produced. Proton and deuteron beams were used to measure key parameters of the system to compare with theoretical estimates. In addition, as a first study case, we present data from the ${ }^{14} \mathrm{~N}(\mathrm{~d}, \alpha){ }^{12} \mathrm{C}$ reaction, at center of mass energies between 1.9 and $3.0 \mathrm{MeV}$ with an $\mathrm{E}-\Delta \mathrm{E}$ telescope detector at $35^{\circ}$. Excitation functions for several excited states were constructed and an ${ }^{16} \mathrm{O}$ resonance at $22.72 \mathrm{MeV}$ was confirmed.
\end{abstract}

DOI: 10.1103/PhysRevSTAB.18.123502

PACS numbers: 07.30.Kf, 29.90.+r

\section{INTRODUCTION}

This paper describes the design, construction and commissioning of a new supersonic gas jet target beam line. It is part of the upgrading program for our 5.5 MV CN-Van de Graaff accelerator facility [1] at Instituto de Física Universidad Nacional Autónoma de México (IFUNAM). This machine has been in operation in Mexico since 1988. The upgrading program of our facility was completed in 2014 and it is now composed of five beam lines, instead of the single previous one dedicated to ion beam analysis (IBA) work. The new additional four beam lines taking into account the development of dedicated instrumentation for: (i) low background gamma-ray detection (astrophysics), (ii) low energy positron beam production (atomic physics), (iii) monochromatic fast neutron beams (nuclear physics) and (iv) SUGAR (an acronym from supersonic gas jet target), which is the subject of this paper. The availability of a pure gas jet target in our low energy beam facility enables a wide range of research projects. Of special interest for our group is the study of nuclear reactions below the Coulomb barrier [2-6] and the production of fast neutron beams with the associated particle technique through $(\mathrm{d}, \mathrm{n})$ reactions [7-10]. With respect to the latter, Table I lists $(\mathrm{d}, \mathrm{n})$ reactions on gas targets with positive $Q$-values.

Published by the American Physical Society under the terms of the Creative Commons Attribution 3.0 License. Further distribution of this work must maintain attribution to the author $(s)$ and the published article's title, journal citation, and DOI.
In the table, ${ }^{2} \mathrm{H}$ and ${ }^{21,22} \mathrm{Ne}$ are rare and expensive substances. ${ }^{3} \mathrm{H}$ is also radioactive so in spite of having the lowest Coulomb barrier and the highest $Q$-value it is not necessarily the best choice to routinely produce fast neutron beams. The large Coulomb barrier on heavy noble gases make them difficult to use in a low energy facility like ours. ${ }^{14} \mathrm{~N}(\mathrm{~d}, \mathrm{n}){ }^{15} \mathrm{O}$ stands as an appealing option to produce fast neutrons in comparison with the traditional $d(d, n)^{3} \mathrm{He}$ reaction using a solid $C D_{2}$ (deuterated polyethylene) target. Atmospheric ${ }^{14} \mathrm{~N}$ is readily available at no cost, and has a larger $Q$-value.

To use a nitrogen supersonic gas jet target (considering a $99.6 \%$ of ${ }^{14} \mathrm{~N}$ natural isotopic abundance) also has all the advantages that windowless gas targets have over solid ones: its thickness and composition remain unaltered even when the beam intensity is large or the time of exposure is long, since target atoms are being changed continuously as the gas flows. There is no buildup of foreign particles on its

TABLE I. $(\mathrm{d}, \mathrm{n})$ reactions on gas targets with positive $Q$-value.

\begin{tabular}{lccc}
\hline \hline Target & Residue & $Q[\mathrm{MeV}]$ & Coulomb E $[\mathrm{MeV}]$ \\
\hline${ }^{2} \mathrm{H}$ & ${ }^{3} \mathrm{He}$ & 3.27 & 0.48 \\
${ }^{3} \mathrm{H}$ & ${ }^{4} \mathrm{He}$ & 17.59 & 0.44 \\
${ }^{14} \mathrm{~N}$ & ${ }^{15} \mathrm{O}$ & 5.07 & 2.29 \\
${ }^{20} \mathrm{Ne}$ & ${ }^{21} \mathrm{Na}$ & 0.21 & 3.02 \\
${ }^{21} \mathrm{Ne}$ & ${ }^{22} \mathrm{Na}$ & 4.51 & 2.99 \\
${ }^{22} \mathrm{Ne}$ & ${ }^{23} \mathrm{Na}$ & 6.57 & 2.95 \\
${ }^{40} \mathrm{Ar}$ & ${ }^{41} \mathrm{~K}$ & 5.58 & 4.62 \\
${ }^{80} \mathrm{Kr}$ & ${ }^{81} \mathrm{Rb}$ & 2.63 & 7.76 \\
\hline \hline
\end{tabular}


surface, meaning no sputtering and hence no structural damage. Systems like this one have been built and tested successfully previously for high energy physics [11-13], high speed electron acceleration [14] as well as nuclear physics $[15,16]$. In addition, supersonic gas jet targets are being developed for nuclear astrophysics experiments $[17,18]$. Jets can also be used for atomic physics [19], and interesting applications in beam diagnostics [20-23] from which medical facilities such as NIRS-HIMAC benefit [24].

Taking advantage of the availability of low energy deuteron beams, we decided to use it in combination with an air jet target to study the ${ }^{14} \mathrm{~N}(\mathrm{~d}, \alpha){ }^{12} \mathrm{C}$ reaction as a first case of study using SUGAR. This reaction has been recently studied $[25,26]$ for $\alpha_{0}$ and $\alpha_{1}$ at backward angles to provide additional information for IBA applications. Older work using a gas cell can be found [27,28].

In Sec. II SUGAR is described in detail, including the experimental setups used for the commissioning runs and the study of the deuteron nuclear reactions on nitrogen.

Data analysis of the ${ }^{14} \mathrm{~N}(\mathrm{~d}, \alpha){ }^{12} \mathrm{C}$ reaction is presented in Sec. III. The summary and conclusions of this work can be found in Sec. IV.

\section{EXPERIMENTAL ARRAY}

\section{A. SUGAR}

A windowless supersonic gas jet target constitutes a big leak for a vacuum system. Accelerated particles are transported in high vacuum $\left(10^{-5-7}\right.$ Torr) from the ion source, through the accelerator and beam lines to the target. Connecting SUGAR to the accelerator beam line requires a differential pumping system; a series of independent pumping stations, interconnected by apertures large enough to allow transmission of beam and as small as possible to minimize the gas throughput from one chamber to the next. The pressure in the chamber that connects SUGAR to the accelerator has to be at least as low as that in the accelerator pipes. A pneumatic valve isolates SUGAR from the accelerator beam line. This valve remains closed until the pressure measured in this chamber is below the pressure in the accelerator beam line.

\section{B. Differential pumping system}

Every beam from the accelerator entering SUGAR has to go through our differential pumping system before reaching the jet chamber. Figure 1 shows a sketch of SUGAR: the differential pumping system consists of three independent consecutive pumping stations. The chambers in each pumping station were designed as identical modules, to be constructed out of standard components of the vacuum industry. Minimizing the work load on our workshop and the time required for assembly. These are six-way LF200 crosses chambers. The one closer to the accelerator has a turbomolecular pump (ALCATEL ATP 400 l/s), while the

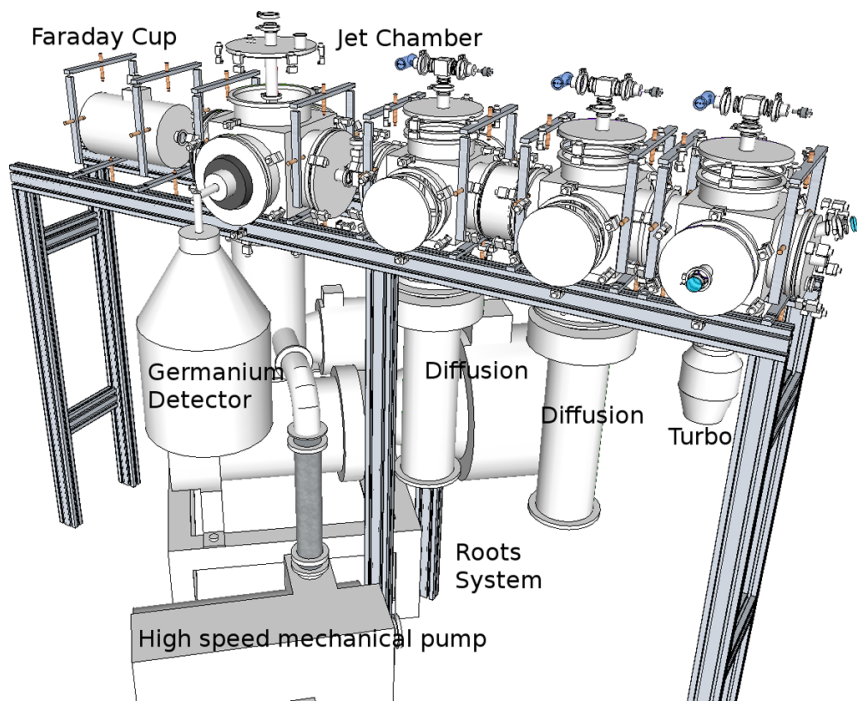

FIG. 1. 3D sketch of SUGAR. The differential pumping system is on the right-hand side towards the accelerator beam line. The scattering chamber (with a HPGe detector) is on the left. The beam line ends in a Faraday cup at the far left.

two closer to the scattering chamber have high speed diffusion pumps (Varian M6 1200 liters/ sec). Every pumping stage pressure can be monitored by either an ion gauge or a thermocouple connected via ISO QF40 adapters. While the pumping chambers are ISO LF200, as we mentioned before, the scattering chamber is an ISO LF250.

The typical pressures attained during operation are 0.1 Torr at the scattering chamber and $10^{-7}$ Torr before connecting to the accelerator vacuum system.

Each chamber in the differential pumping system of SUGAR is isolated from the next one except for a $5 \mathrm{~mm}$ circular aperture. These apertures are all made the same: a LF200 flange with a centering ring assembly in both sides and a QF40 nipple in their center. Figure 2 shows this arrangement schematically.

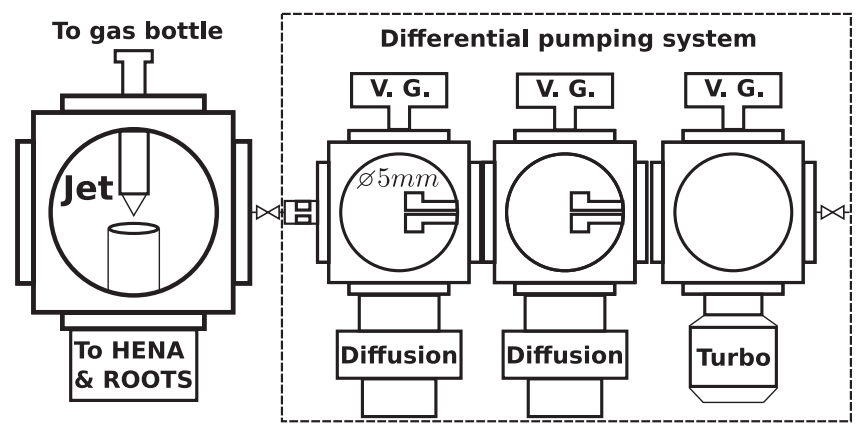

FIG. 2. 2D Schematic view of SUGAR where the three $5 \mathrm{~mm}$ diameter apertures can be seen: two of them inside of each of the two middle chambers [each of these have also a vacuum gauge (V.G.)], whereas the third one connects the differential pumping system to the scattering chamber. The V.G.'s are employed to limit the gas throughput and still allow the beam to reach the jet. 
The design of SUGAR with standard vacuum elements in addition, is conducive to upgrades and further modifications. Pumping speed can be significantly improved with the installation of additional pumps in the LF200 flanges.

A significant effort was devoted to perfectly align these apertures to maximize transmission of the beam from the switching magnet all the way to the gas target. After an optical alignment, beam transmission was optimized using two Faraday cups. One is shown in Fig. 1 and a second one (not shown) is placed at the exit of the switching magnet, just after the beam profile monitor.

It is worth mentioning at this point that the design of our differential pumping system (pumping speeds, aperture sizes, volumes, etc.) followed the results of detailed gas flow calculations. To perform such calculations we wrote a custom computer code [29] following the theory described in the next section.

\section{Scattering-jet chamber and nozzle}

The scattering-jet chamber is a six-way LF250 cross type. It is separated from the differential pumping system by a QF40 gate valve. This allows for modifications in the experimental setup without the need to shut down the differential pumping system. A high speed mechanical pump (60 l/s Pfeiffer Hena 200) is connected to the central region of the scattering chamber (the catcher). This pump removes most of the gas coming out from the nozzle (the upper part that completes our system). Gas spilling out of the catcher into the scattering chamber is pumped out from the bottom (via the LF250 flange) by a large $(13 \mathrm{~kW})$ high speed roots pump (Pfeiffer WKP 4000 AM, 1400 l/s), backed up by a second roots pump (Pfeiffer WKP 1000 $\mathrm{AM}, 350 \mathrm{l} / \mathrm{s}$ ) which in turn is backed up by a mechanical pump (Pfeiffer Hena 60, 20 1/s). Once the pressure in the scattering chamber is around $10^{-1}$ Torr, the valve between the scattering chamber and the differential pumping system can be opened.

For experiments requiring gamma ray detection, the scattering chamber allows for a special LF250 (nose) flange as sketched in Fig. 1. This device serves to accommodate an external $\mathrm{HpGe}$ detector as close as possible to the target.

The supersonic gas jet target is produced by injecting a high pressure gas (1-5 atm) into the scattering chamber (Fig. 3) at a low vacuum ( $10^{-1}$ Torr) through a special nozzle whose shape forces a supersonic flow. This gas must be evacuated as soon as it enters in the scattering chamber. Its vacuum system consists of two pumping stages: an inner one that takes most of the injected gas [the catcher in Fig. 4] and an outer one to pump out the rest.

Given the geometry of the nozzle and the pressure at the entrance, we can calculate flow properties such as the Mach number $(M)$ and the expansion angles (via the PrandtlMeyer function). The theory for this calculation can be found in Ackroyd and Pritchard books [30,31]. The Mach

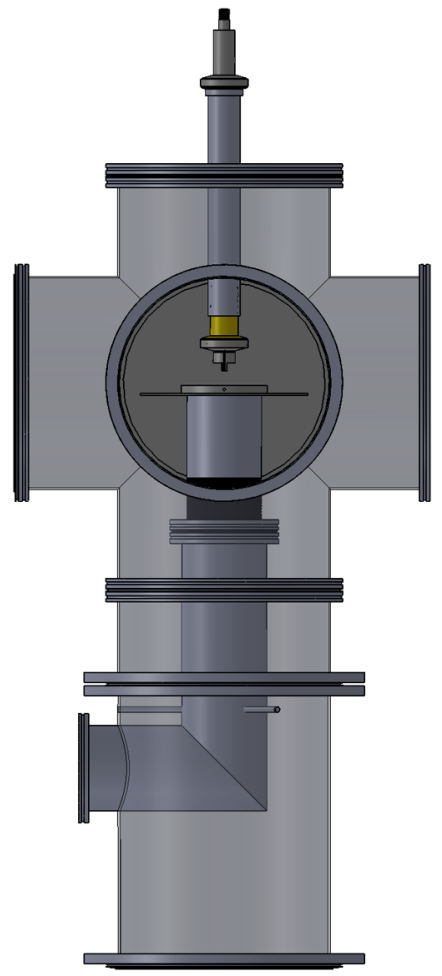

FIG. 3. Sketch of the scattering chamber details. The figure shows the inner piping of the LF250 scattering chamber, the top part of the inner pipe is the catcher. It is connected to a threaded LF100 flange in its middle section for varying the distance to the nozzle. A table can be attached to it for placing detectors inside.

number can be obtained from the throat to exit area ratio through the isentropic flow equation:

$$
\frac{A}{A^{*}}=\frac{1}{M}\left[\frac{1+\frac{\gamma-1}{2} M^{2}}{(\gamma+1) / 2}\right]^{\frac{\gamma+1}{2(\gamma-1)}},
$$

where $A^{*}$ is the nozzle throat area, $A$ is the exit area and $\gamma$ is the heat capacity ratio $(\mathrm{Ce} / \mathrm{Cv})$ (1.4 for nitrogen). For instance a ratio area of 10 corresponds to a Mach $(M)$ number near to 4 .

In Fig. 4, $\alpha$ is the opening angle between the nozzle input and output $\left(26^{\circ}\right)$. This is a characteristic of each nozzle. The angle $\theta\left(64^{\circ}\right)$ describes the spill of the jet. The first shock zone, "the tongue" where the beam will hit, can be approximated by this triangular region characterized by the angle $\mu\left(14^{\circ}\right)$, its height $e(6 \mathrm{~mm})$, and base $d(2.8 \mathrm{~mm})$. Finally $H$ is the distance from the nozzle to the catcher $(2 \mathrm{~cm})$, which can be varied since both, the connections of the nozzle and the catcher, are threaded. We can extract the throughput from the nozzle into the scattering chamber through the mass flow rate equation [30]:

$$
\dot{m}=\gamma^{\frac{1}{2}}\left[\frac{\gamma+1}{2}\right]^{-\frac{\gamma+1}{2(\gamma-1)}}\left(p_{0} \rho_{0}\right) A^{*},
$$

where the nozzle throat area $A^{*}=2 \times 10^{-2} \times 7.5 \times$ $10^{-1} \mathrm{~cm}^{2}=1.5 \times 10^{-2} \mathrm{~cm}^{2}$ and $p_{0}, \rho_{0}$ are the input 


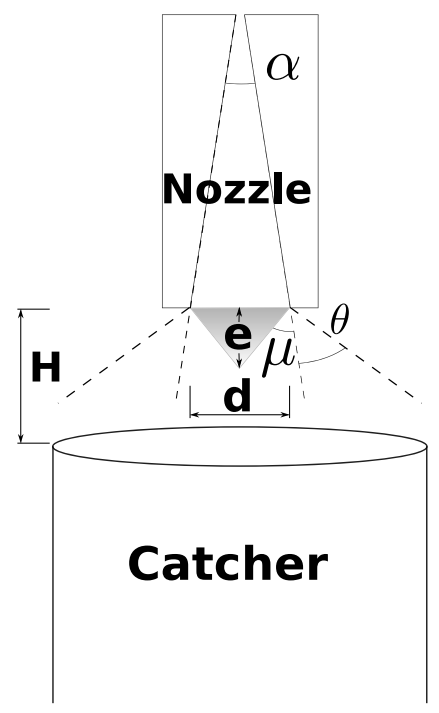

(a)The nozzle and its catcher.
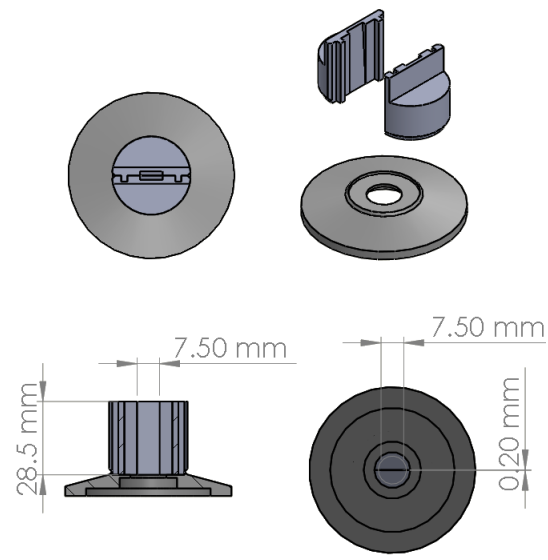

(b)Design of a rectangular nozzle.

FIG. 4. (a) Layout of the nozzle and its catcher (see the text for details). (b) Construction sketches of the nozzle pieces with dimensions.

pressure and density respectively. In order to express the pressure in Torr, the density in $\mathrm{g} / \mathrm{cm}^{3}$, and the area in $\mathrm{cm}^{2}$ we have to multiply it by $(133.32)^{1 / 2} \times 10=36.5$. For the case of nitrogen at atmospheric pressure $\left(p_{0}=760\right.$ Torr, $\rho_{0}=10^{-3} \mathrm{~g} / \mathrm{cm}^{3}$ ) we have an approximate mass flow rate $\dot{m}=0.17 \mathrm{~g} / \mathrm{s}$. The throughput $q_{m}$ into the chamber will be

$$
q_{m}=\frac{\dot{m}}{\text { molweight }}(760 \times 22.4) \text { Torr } 1,
$$

where 760 and 22.4 in Eq. (3) are pressure and volume respectively at standard conditions. The molecular weight for nitrogen is $28 \mathrm{~g} /$ mole giving a $q_{m}=10^{2}$ Torr $1 / \mathrm{s}$. It is important to note that for supersonic flow, the Mach number of the flow determines partially the density following the relationship of the isentropic flow equation for density [30]. Expressing it in terms of particle number,

$$
n=n_{0}\left[1+\frac{\gamma-1}{2} M^{2}\right]^{-\frac{1}{\gamma-1}},
$$

where $n_{0}$ and $n$ are the reservoir and exit densities respectively. Substituting our obtained values we get $n \approx 7 \times 10^{17}$ molecules $/ \mathrm{cm}^{3}$, considering molecular nitrogen $\left(\mathrm{N}_{2}\right)$ and multiplying by the nozzle thickness $(0.75 \mathrm{~cm})$ we get the areal density of $10^{18}$ particles $/ \mathrm{cm}^{2}$. From the spilling cone we calculate the throughput into the rest of the system (10\% spill is a good estimation). For the central region we cannot use Eq. (3) to estimate the pressure at which the pump will be working. This pressure is actually defined by the stagnation condition of the jet flow downstream, after the shocks. Here we must use the RankineHugoniot relations. Ackroyd [30] and Liepman [32] have explained thoroughly the procedure. We constructed our nozzle with a rectangular shape: $28.5 \mathrm{~mm}$ long, with 0.2 and $2.8 \mathrm{~mm}$ for throat and exit aperture heights and $7.5 \mathrm{~mm}$ width in both cases [as shown in Fig. 4(b)]. Machining tolerances are all estimated in $10 \%$. Such a rectangular nozzle allows us to have a variable thickness target by simply rotating the nozzle.

The system allows for the use of multiple nozzle types. The nozzle throughput can be changed with its geometry. Figure 4(b) shows the design and assembly of one of the rectangular nozzles. A QF40 blank flange was modified in order to accommodate the rectangular stainless steel nozzle. Much of the material was scrapped off the nozzle exit in order to minimize beam interaction with the nozzle material.

Figure 5 shows the modified LF250 flange in order to connect the nozzle to the system, overpressure rings are needed in order to avoid the gas from leaking through the

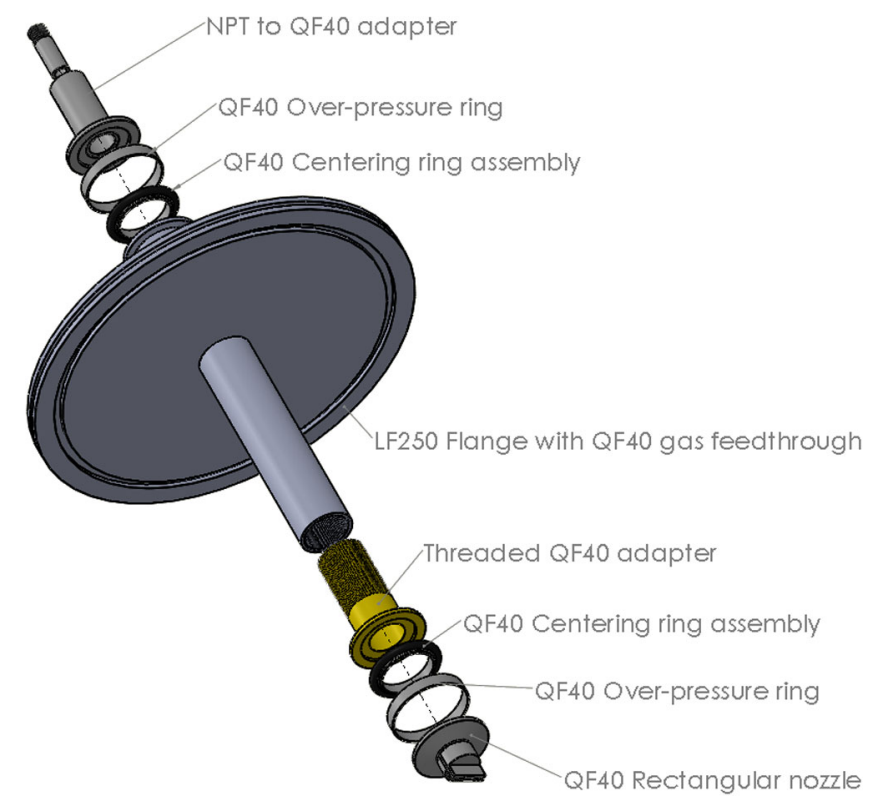

FIG. 5. Exploded view of the nozzle connection to the system. Wing nut clamps are not shown. 
vacuum seal. The threaded QF40 adapter allows for an adjustable height of the nozzle position and also simplifies the rotation of the target (for the variable thickness feature). The adapter was made out of brass, the threads in the LF250 flange are stainless steel, preventing potential problems with the pieces getting stuck. Teflon was used, limiting the leaks through the threads.

This throughput can be augmented up to an order of magnitude by increasing the pumping speed in the differential system. This can be easily achieved due to the modular design of the system that allows to attach extra pumps.

The nozzle can be connected to the gas bottle through the NPT to QF40 adapter as in Fig. 5 or directly, for testing the nozzle outside the system.

\section{Operation of SUGAR}

\section{Pressures}

The first pressure test for the proper operation of SUGAR was done by measuring the pressures in different points along the system and comparing them to the theoretical predictions. All pressure measurements were made with Varian type 0531 vacuum gauges, the ion gauges of Duniway stockroom Corporation T-075-K and Varian K2471302. Some of the calculations were overestimated in order to account for nonideal behaviors such as unexpected leaks. Figure 6 (top left) depicts the evolution of the pressure as a function of time for the pumping down of the system with a mechanical pump. The ideal exponential fall is only present for a brief period of time (top right). This behavior is reproducible as can be seen by the second data take (blue curve) in the bottom part of Fig. 6. The sudden drop of the curve (around $3500 \mathrm{~s}$ ) is due to the

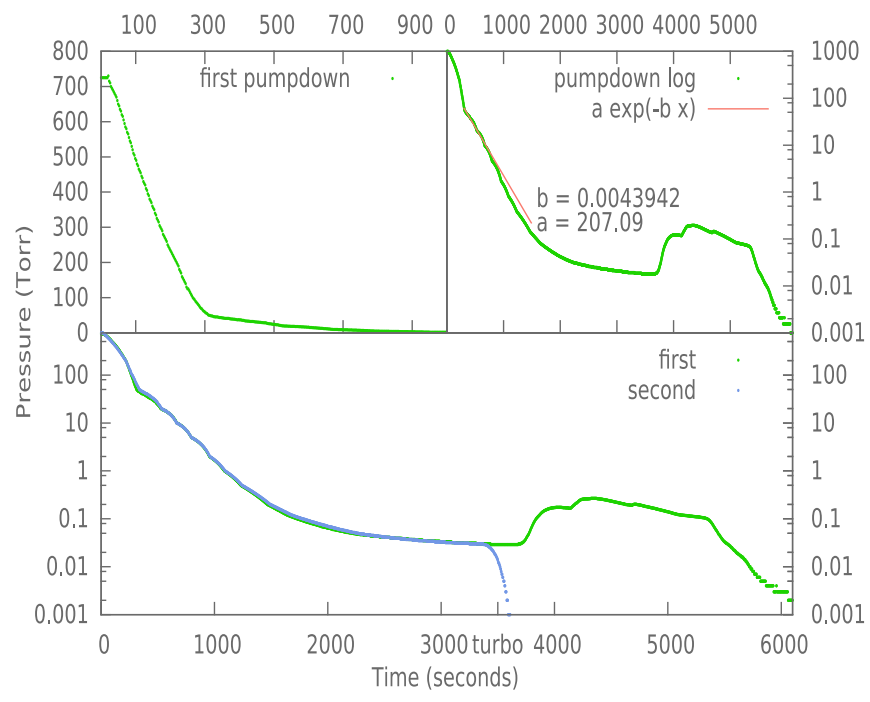

FIG. 6. Evolution of the pressure in the turbo chamber as a function of the time for SUGAR system (see the text for details).
TABLE II. Calculated vs measured pressures (for air). All values are given in Torr.

\begin{tabular}{lcc}
\hline \hline Chamber & $P_{\text {calculated }}[$ Torr] & $P_{\text {measured }}[$ Torr] \\
\hline Scattering & 0.1 & 0.115 \\
Diffusion 2 & $8.0 \times 10^{-4}$ & $2.0 \times 10^{-4}$ \\
Diffusion 1 & $1.8 \times 10^{-6}$ & $1.0 \times 10^{-5}$ \\
Turbo & $2.0 \times 10^{-8}$ & $1.6 \times 10^{-6}$ \\
\hline \hline
\end{tabular}

turbomolecular pump being turned on, which shows on its initial stage also an exponential decrease.

Table II shows a comparison between the calculated and measured pressures for a jet of air injected at atmospheric pressure. Calculations take into account geometry and throughputs of the system.

Predicted and measured values do match in the scattering chamber however a discrepancy starts to show towards the turbo chamber, see Table II. We believe this discrepancy is mostly due to unaccounted leak sources and nonideal behavior of the vacuum pumps in the calculations.

\section{E. The Schlieren technique}

Some of the nozzles were tested outside the system, in a fluids laboratory, for shock pattern structure visualization of the supersonic gas jet flow. Figure 7 shows the results of these tests for illustration purposes.

The Schlieren technique is used to visualize transparent flows that cannot be seen by the human eye without aid. It uses the relationship between refraction index and density. The visualization is done with an optical system with lenses and an attenuator. For the case shown in Fig. 7, the air jet was illuminated with a laser of $150 \mathrm{~mW}$ and $532 \mathrm{~nm}$ wavelength (green). Figure 7(b) shows the optical setup for the Schlieren technique. We used two lenses with respective focal lengths of 18 and $20 \mathrm{~cm}$. Because the light source was coherent, an attenuator FSD-100 was used as a filter instead of a knife edge. A nozzle with a straight cylindrical shape (1.5 mm diameter) was used in this visualization experiment. The Mach number is defined as the ratio between the local flow speed and the local speed of sound: $M=v / c$. It can also be obtained from the angle defined in the shock patterns through $M=1 / \sin (\alpha)$ [31], where the angle alpha is the small angle in the shock region [Fig. 7(a)]. We were able to measure the Mach number through these angles off the $\mathrm{X}$ patterns in the images giving: $M=2.1 \pm 14 \%, 2.4 \pm 13 \%, 2.8 \pm 12 \%$ for pressures $p=2.5,2.8,3.7$ atm, respectively.

In the leftmost two images of Fig. 7(a), the inlet to exit pressure ratios are not high enough to produce shock patterns. In the rightmost three photographs (the highest pressures), the pressure differences are too big, thus we are unable to determine the Mach number following the same procedure. Figure 7 also shows [in (b)] the typical structure of supersonic flows; even though the gas is moving fast, the 


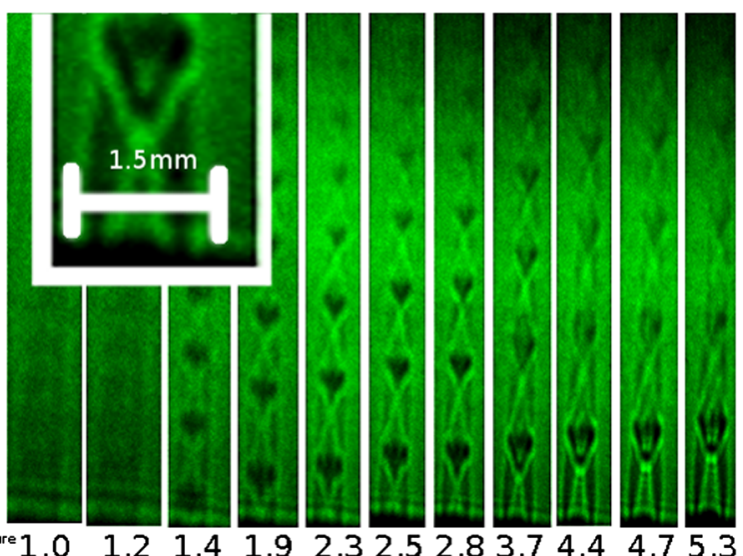

$\begin{array}{llllllllllll}\substack{\text { Pressure } \\ \text { (atm) }} & 1.0 & 1.2 & 1.4 & 1.9 & 2.3 & 2.5 & 2.8 & 3.7 & 4.4 & 4.7 & 5.3\end{array}$

(a)Schlieren images of the jet at different pressures, indicated under each flow.

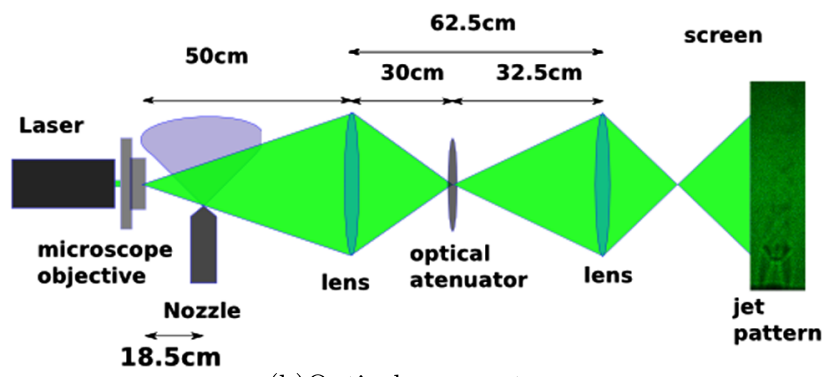

(b)Optical array setup.

FIG. 7. (a) Schlieren photographic images obtained using a circular nozzle. Each vertical strip corresponds to the pattern observed when air is forced upwards into the atmospheric pressure of the laboratory. The corresponding value of the injection pressure for each photograph is labeled in the bottom (atm units). (b) Optical array: in this case a simple rectangular aperture was used. The flow goes from an air bottle at high pressure into the atmosphere.

patterns remain stationary. Such patterns occur multiple times downstream.

These results prove that the Schlieren technique is appropriate to monitor continuously supersonic gas flows. Its implementation to monitor gas flows within SUGAR is in progress.

\section{F. Characterization run}

A direct test of the jet target itself was made injecting air at atmospheric pressure (using the rectangular nozzle) and bombarding it with $1.8 \mathrm{MeV}$ protons. Beam current on target was $0.5 \mu \mathrm{A}$. A surface barrier detector was placed at $\theta_{\text {Lab }}=165^{\circ}$. Figure 8 shows a typical spectrum. Three well-defined peaks were identified, which correspond to elastic scattering of protons with nitrogen, oxygen and argon. The SIMNRA [33] computer code was used to simulate this spectrum. This was further verified by performing pure nitrogen and pure argon experiments, the data is superimposed with the air data (Fig. 8). Proton elastic cross sections at this bombarding energy are not Rutherford. Instead elastic backscattered (EBS)

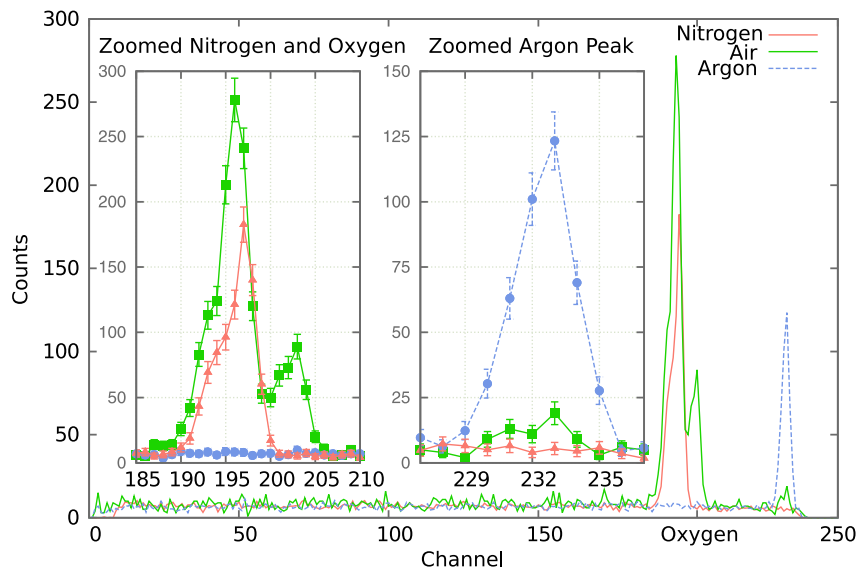

FIG. 8. The spectrum of scattered particles from a $1.8 \mathrm{MeV}$ proton beam on the air supersonic gas jet. The surface barrier detector was set at a $165^{\circ}$ angle. The regions of interest (for nitrogen, oxygen and argon) are shown in the center part of the plot along with the statistical error bars.

cross sections (IBANDL [34]) were used. With this, elemental concentrations were deduced to be $74.5 \%$, $23.3 \%$ and $2.2 \%$ respectively for $\mathrm{N}, \mathrm{O}$ and Ar. These values are comparable with the typical abundances reported [35]: $78.08 \%, 20.95 \%$ and $0.98 \%$.

A very important value that could be obtained from the SIMNRA simulation is the target thickness (i.e. an estimated density). What came out for an atmospheric pressure injection resulted to be around $10^{18}$ atoms $/ \mathrm{cm}^{2} \pm 10 \%$. This is a good enough thickness to develop good precision experiments and it matches the estimate given in Sec. II C. Jet density was also measured with various bottle pressures and is presented in [36]. Other jet densities go from $10^{17}$ atoms $/ \mathrm{cm}^{2}$ [37] to $10^{19}$ atoms $/ \mathrm{cm}^{2}$ [38].

\section{G. Deuteron induced reactions on an air jet}

The experimental array inside the scattering chamber is shown in Fig. 9. A $1 \mathrm{~cm}$ long $3 \mathrm{~mm}$ diameter collimator was placed in front of a telescopic array of silicon detectors at $35^{\circ}$ with respect to the beam. This long collimator stops particles produced by beam interactions with residual gas

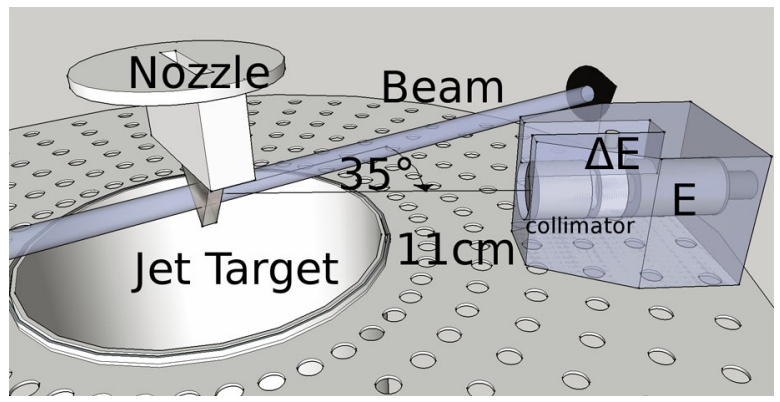

FIG. 9. Experimental setup. Inside the scattering chamber, a collimated $\mathrm{E}-\Delta \mathrm{E}$ telescope array was placed at $35^{\circ}$ with respect to the beam axis, in order to measure forward reactions and elastic scattering. 
molecules in its path before or after the high density jet target. A very thin ORTEC planar detector $(11 \mu \mathrm{m}, 9 \mathrm{~V})$ was used as $\Delta \mathrm{E}$ and a thicker $(300 \mu \mathrm{m}, 60 \mathrm{~V})$ CANBERRA PIPS detector as E. Light particles punch through the detector and stop in the $\mathrm{E}$ detector. Particle identification can be then carried out by the well-known E- $\Delta \mathrm{E}$ technique. Heavy ions are stopped by the $\Delta \mathrm{E}$ detector where no positive identification is possible, although some groups can be partially identified by its energy from kinematics.

A standard triple alpha source $\left({ }^{239} \mathrm{Pu},{ }^{241} \mathrm{Am},{ }^{244} \mathrm{Cm}\right.$ : $E_{\alpha}=5.15,5.48$ and $5.8 \mathrm{MeV}$, respectively) was used to calibrate in energy both elements of our telescope. Once the $\mathrm{E}$ detector is calibrated, the energy loss in the $\Delta \mathrm{E}$ detector for each alpha energy is measured $(2.55,2.41$ and $2.29 \mathrm{MeV}$ respectively) to calibrate the transmission detector.

The gas jet target was produced by injecting air at atmospheric pressure (78\% nitrogen, 20\% oxygen and 2\% other elements).

Deuteron energies were varied between 2.2 and 3.36 MeV. Beam intensities were measured in a Faraday cup before the scattering chamber during beam setup and optimized before data taking. Typical beam currents were between 200 and $1000 \mathrm{nA}$. Signals from the silicon detector array were handled by standard nuclear instrumentation module electronics.

Data acquisition ran in a PC with a Windows platform. The control program has been developed with LABVIEW software. A computed automated measurement and control interfaces the PC and the detection system. In this experiment the trigger was provided by the $\Delta \mathrm{E}$ detector signals. The logic signal was produced by a discriminator keeping the threshold just above the electronic noise to include low energy signals.

\section{RESULTS}

The ${ }^{14} \mathrm{~N}+\mathrm{d}$ reaction has several exit channels with relatively high $Q$ values (Table III). The Coulomb barrier is around $2.3 \mathrm{MeV}$ to form the ${ }^{16} \mathrm{O}$ compound nucleus. Since the $Q$-value for fusion is very large $(20.74 \mathrm{MeV})$ we are able to study a high excitation region in ${ }^{16} \mathrm{O}$.

In this paper we concentrate on the ${ }^{14} \mathrm{~N}(\mathrm{~d}, \alpha){ }^{12} \mathrm{C}$ channel where alpha particles are detected at a fixed angle in the

TABLE III. There are only seven channels open for beam energies below $5 \mathrm{MeV}$.

\begin{tabular}{lcc}
\hline \hline Ejectile & Residual & Q [MeV] \\
\hline$\gamma$ & ${ }^{16} \mathrm{O}$ & 20.74 \\
$\alpha$ & ${ }^{12} \mathrm{C}$ & 13.57 \\
$p$ & ${ }^{15} \mathrm{~N}$ & 8.61 \\
$4 \alpha$ & $\ldots$ & 6.29 \\
$n$ & ${ }^{15} \mathrm{O}$ & 5.07 \\
$d$ & ${ }^{14} \mathrm{~N}$ & 0 \\
${ }^{3} \mathrm{He}$ & ${ }^{13} \mathrm{C}$ & -2.06 \\
${ }^{3} \mathrm{H}$ & ${ }^{13} \mathrm{~N}$ & -4.30 \\
\hline \hline
\end{tabular}

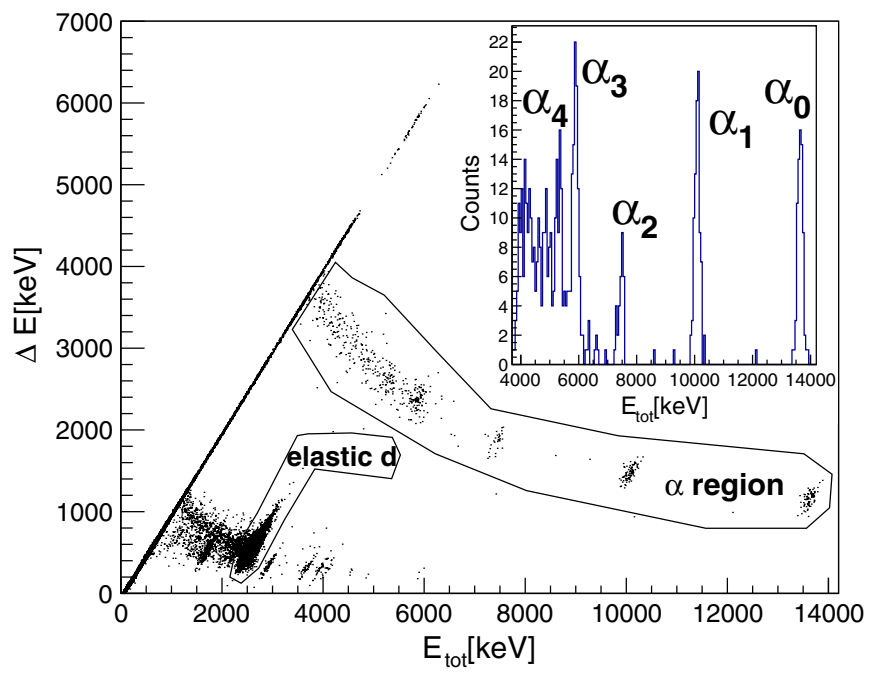

FIG. 10. Typical two-dimensional histogram $\Delta \mathrm{E}$ vs $E_{\text {tot }}$ of the $\mathrm{d}+{ }^{14} \mathrm{~N}$ reaction $\left(E_{c m}=2.51 \mathrm{MeV}\right)$. Deuterons and alphas are clearly identified. The elastic peak is used in each case to extend and confirm the energy calibration and for normalization purposes (see text). In the subframe, a projection from the total energy spectrum of the $\alpha$ banana is shown.

laboratory, while the bombarding energy is changed between 2.2 and $3.36 \mathrm{MeV}$. So our study spans an energy interval right at and above the top of the barrier.

Data reduction and analysis was done with the software ROOT [39]. All histograms shown here (1 and 2d) were generated with it. A typical two-dimensional histogram from our data is shown in Fig. 10. In the vertical axis we plot the energy calibrated signal from the $\Delta \mathrm{E}$ detector and in the horizontal axis, the calibrated total energy recorded by our telescope $E_{\mathrm{tot}}=\mathrm{E}+\Delta \mathrm{E}$. Elastically scattered deuterons were used to verify the energy calibration of the beam.

As can be seen in Fig. 10, several alpha groups are present and can be identified as emission from the compound nucleus ${ }^{16} \mathrm{O}$ where the ${ }^{12} \mathrm{C}$ lays in different excited states. Table IV gives the energy expected for each alpha group in our detector $\left(35^{\circ} \mathrm{lab}\right)$ as a function of beam energy. The corresponding ${ }^{12} \mathrm{C}$ excitation energies as well as the center of mass energy are also given.

Values in Table IV were calculated from known ${ }^{12} \mathrm{C}$ excited states (row 2). Note that in the subplot of Fig. 10, the alpha peaks match (better than 3\%) the expected energies in Table IV. Similar agreement was observed for all beam energies.

Since the elastically scattered particles and the nuclear reaction products share the same beam intensity, target density and solid angle, it can be easily shown that the alpha cross section can be computed using the following equation:

$$
\left(\frac{d \sigma_{\alpha}}{d \Omega}\right)_{\theta_{\mathrm{Lab}}}=\frac{N_{\alpha}}{N_{d}}\left(\frac{d \sigma_{\mathrm{El}}}{d \Omega}\right)_{\theta_{\mathrm{Lab}}}
$$


TABLE IV. Center of mass energies and the corresponding alpha energies for the different ${ }^{12} \mathrm{C}$ excitation levels for all deuteron beam energies ran in this experiment. The values in bold correspond to Fig. 10. Note: All energies are in MeV.

\begin{tabular}{|c|c|c|c|c|c|c|}
\hline & $\begin{array}{c}\text { Carbon } \\
\text { Excitation } \\
\text { Energy }[\mathrm{MeV}]\end{array}$ & $\begin{array}{c}\text { Base } \\
0\end{array}$ & $\begin{array}{l}\text { First } \\
4.43\end{array}$ & $\begin{array}{c}\text { Second } \\
7.65\end{array}$ & $\begin{array}{l}\text { Third } \\
9.65\end{array}$ & $\begin{array}{c}\text { Fourth } \\
10.3\end{array}$ \\
\hline$E_{L a b}$ & $E_{c m}$ & $\alpha_{0}$ & $\alpha_{1}$ & $\alpha_{2}$ & $\alpha_{3}$ & $\alpha_{4}$ \\
\hline 2.20 & 1.92 & 13.11 & 9.55 & 6.94 & 5.31 & 4.76 \\
\hline 2.29 & 2.01 & 13.20 & 9.64 & 7.03 & 5.4 & 4.8 \\
\hline 2.40 & 2.1 & 13.31 & 9.75 & 7.13 & 5.50 & 4.95 \\
\hline 2.50 & 2.19 & 13.42 & 9.85 & 7.23 & 5.59 & 5.04 \\
\hline 2.61 & 2.29 & 13.53 & 9.96 & 7.33 & 5.14 & 5.14 \\
\hline 2.73 & 2.39 & 13.65 & 10.07 & 7.45 & 5.81 & 5.26 \\
\hline 2.87 & 2.51 & 13.79 & 10.21 & 7.58 & 5.94 & 5.38 \\
\hline 2.97 & 2.6 & 13.89 & 10.31 & 7.68 & 6.03 & 5.48 \\
\hline 3.10 & 2.71 & 14.02 & 10.43 & 7.8 & 6.15 & 5.6 \\
\hline 3.26 & 2.85 & 14.18 & 10.59 & 7.95 & 6.3 & 5.74 \\
\hline 3.36 & 2.95 & 14.28 & 10.68 & 8.07 & 6.39 & 5.83 \\
\hline
\end{tabular}

which directly links the elastic scattering cross section to the nuclear reaction of interest for each corresponding energy region. This relation makes the normalization of the data for the cross section calculation very direct. $N_{\alpha}$ and $N_{d}$ stand for the number of alphas and deuterons reaching the detector, and $\left(\frac{d \sigma_{\mathrm{El}}}{d \Omega}\right)_{\theta_{\mathrm{Lab}}}$ stands for the elastic scattering differential cross section at the same angle and energy, in the laboratory reference frame. Figure 11 shows the experimental excitation functions, where the cross section of the various alphas are shown in terms of the center of mass energy.

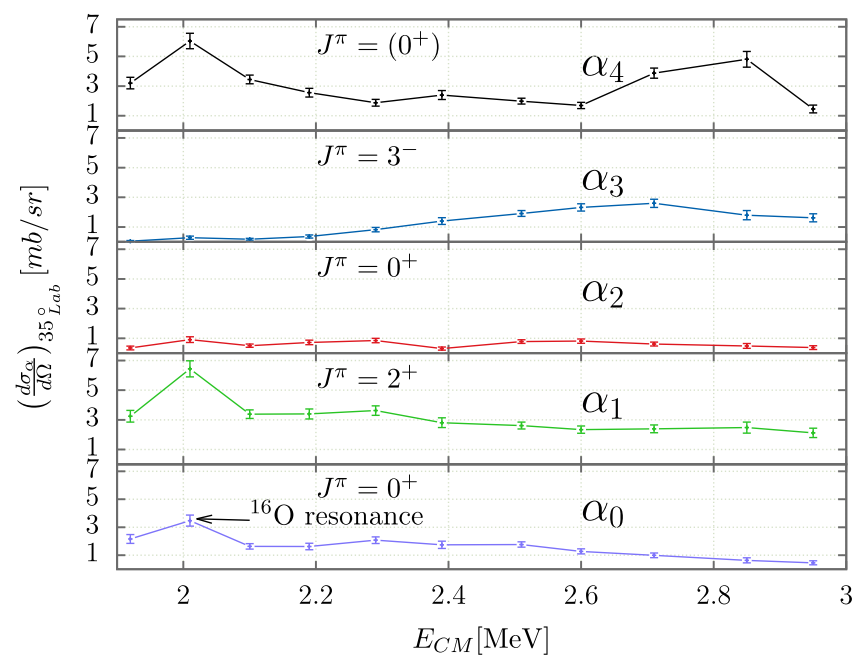

FIG. 11. Differential cross section at $35^{\circ}$ in the laboratory system for alpha decays to the lowest lying ${ }^{12} \mathrm{C}$ nuclear states, statistical error bars are also shown (the average error is around $15 \%$ with a minimum of $8 \%$ and maximum of $35 \%$, no systematic errors are reported). Quantum numbers (spin and parity) of each state are given. The lines are drawn to guide the eye.

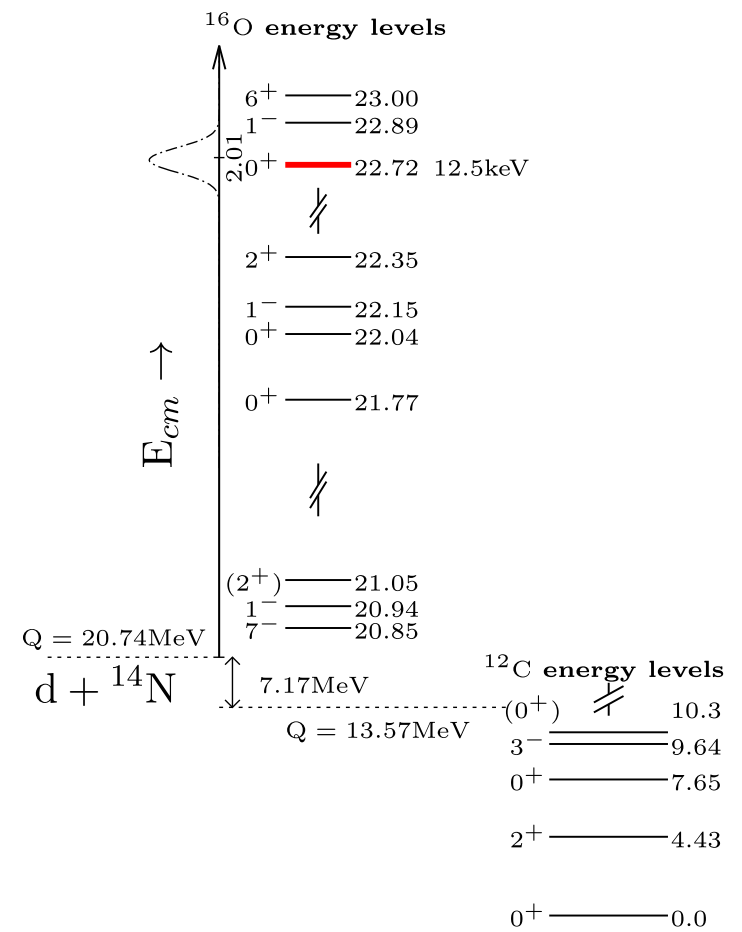

FIG. 12. ${ }^{16} \mathrm{O}$ nuclear level scheme. On the left, the entrance channel $\left(\mathrm{d}+{ }^{14} \mathrm{~N}\right)$ is marked. On the right, $\alpha$ emission to different excited states in ${ }^{12} \mathrm{C}$ is also shown.

A common maximum is found in all cross sections at $E_{c m}=2.01 \mathrm{MeV}$ which corresponds to an ${ }^{16} \mathrm{O}$ resonance around $22.72 \mathrm{MeV}$ (Fig. 12). We also notice that $\alpha_{1}$ and $\alpha_{4}$ have, on average, the highest cross sections and that around $2.8 \mathrm{MeV}$ the $\alpha_{4}$ is the largest. In this respect, it is worth pointing out that nearly $20 \%$ of the air's composition is ${ }^{16} \mathrm{O}$. The ${ }^{16} \mathrm{O}(\mathrm{d}, \alpha){ }^{14} \mathrm{~N}$ has a $Q=3.11 \mathrm{MeV}$. The $\alpha_{0}$ for this reaction can overlap with the $\alpha_{4}$ from our reaction (Table IV) and this can affect our cross section measurement. A pure nitrogen target should be used to verify this point.

\section{SUMMARY AND CONCLUSIONS}

In this paper, SUGAR is described in all its parts. It has been designed to take advantage of parts that can be found commercially, decreasing considerably the need for special parts and adaptors custom made in a mechanical shop. Spare parts and extensions to SUGAR can then be found and installed very easily.

Due to the large target thickness achieved with this system, SUGAR is an ideal instrument to perform high precision cross section measurements with gaseous targets. Particularly when these cross sections are very small, as required for nuclear astrophysics (nucleosynthesis, stellar evolution etc.) considering that supersonic gas jet targets can withstand very large beam intensities without modifying its properties. 
We report here the simple characterization runs with proton beams. Elastic backscattering data $\left(165^{\circ} \mathrm{lab}\right)$ collected on supersonic jets of air, nitrogen and argon are collected. Air spectra were simulated with the help of the code SIMNRA, producing abundances in good agreement with the expected values. Areal densities around $10^{18} \mathrm{part} / \mathrm{cm}^{2}$ (rectangular nozzle) were deduced for inlet pressure of just one atmosphere. All characteristics of the targets were in good agreement with the previously calculated or expected values.

As a first case study using SUGAR, we took data from the ${ }^{14} \mathrm{~N}(\mathrm{~d}, \alpha){ }^{12} \mathrm{C}$ reaction around the Coulomb barrier. This is one of the most prolific reactions induced by deuterons on an air supersonic gas jet target. We extracted excitation functions for $\alpha_{0}, \alpha_{1}, \alpha_{2}, \alpha_{3}$ and $\alpha_{4}\left(35^{\circ} \mathrm{lab}\right)$. The behavior of these excitation functions is consistent with the presence of a previously reported excited state of ${ }^{16} \mathrm{O}$ $\left(0^{+}\right.$at $\left.22.72 \mathrm{MeV}\right)$. It is worth mentioning that previously published work on this reaction is scarce or nonexistent, especially for the $\alpha_{3}$ and $\alpha_{4}$ at forward angles at the energies we report here. We plan to pursue these measurements both with pure nitrogen and air jets as well as with solid thin $\left(\mathrm{Si}_{3} \mathrm{~N}_{4}\right)$ targets. Hauser-Feschbach calculations will be made to understand the relative intensities of the alpha decays observed.

Air gas targets are quite practical: they never run out (no bottle to change), no need to recover at the exhausts of the pumping systems or dumping pure gases into the environment, provide a well-known target composition (N-O-Ar) always fresh and immutable, it is perfect for setup and fine-tuning of the system, there is even a variety of research problems to work with and it is free. For any other kind of target, a bottle is needed. For higher areal density targets, we produced and characterized jets with inlet pressures of up to 5 bar of pure nitrogen and argon from gas bottles.

\section{A. Future work}

A test using the Schlieren technique was made outside the system to visualize the supersonic flow produced by our nozzles. The stationary shock patterns of the flows were shown. We were able to measure Mach numbers at different pressures. Following these successful tests, a similar setup is being designed and tested to monitor the supersonic gas jet targets used in future experiments.

The recuperation system for the gases is still to be implemented. That would allow us to produce a larger variety of targets. Some gases are toxic or explosive, however the leading reasons to have a system that would recover the gas just used as target are the cost and the availability. Week-long experiments on isotopically enriched gas targets $\left({ }^{2} \mathrm{H}\right.$ or ${ }^{3} \mathrm{He}$, of special interest for nuclear astrophysics in capture cross section measurements) are unfeasible without a recuperation system.
The development of tagged fast neutron beams with SUGAR through the ${ }^{14} \mathrm{~N}(\mathrm{~d}, \mathrm{n}){ }^{15} \mathrm{O}$ reaction is an interesting option for the facility and it is already on its way.

A variety of nozzles including Laval shape types have been designed and are under construction. With them, different target shapes and densities will be achieved. For those experiments where the localization of the target has paramount importance, our large nozzle with a rectangular cross section might not be the best suited.

\section{ACKNOWLEDGMENTS}

Professor Dan Shapira's support, encouragements, patience and deep knowledge of every secret needed to build a successful supersonic gas jet target were of paramount importance for this work. Also, the help of Yadira Salazar and Catalina Stern for the optical Schlieren setup was invaluable. Special thanks go to Marco Veytia, Roberto Gleason, Hesiquio Vargas, Carlos Canto and IFUNAM workshop. The authors are grateful to Dr. J. D. Powers for his detailed review and correction of the English of this manuscript. This work was done thanks to the support of CONACYT 51600, 82692,123655 and DGAPAUNAM IN118310, IN-112609, IN-105510, IN-115213, TA-100213, IN-103312.

[1] E. Andrade, M. Feregrino, E. Zavala, J. Pineda, R. Jiménez, and A. Jaidar, Nucl. Instrum. Methods Phys. Res., Sect. A 287, 135 (1990).

[2] P. Rosales and E. Aguilera, Rev. Mex. Phys. 49, 88 (2003).

[3] L. Barrón-Palos and E. Chávez, Rev. Mex. Phys. 50, 18 (2004).

[4] L. Barrón-Palos, E. Chávez, A. Huerta, M. Ortiz, G. Murillo, E. Aguilera, E. Martínez, E. Moreno, R. Policroniades, and A. Varela, Eur. Phys. J. A 25, 645 (2005).

[5] E. F. Aguilera, P. Rosales, E. Martínez-Quiroz, G. Murillo, M. Fernández, H. Berdejo, D. Lizcano, A. GómezCamacho, R. Policroniades, A. Varela, E. Moreno, E. Chávez, M. E. Ortiz, A. Huerta, T. Belyaeva, and M. Wiescher, Phys. Rev. C 73, 064601 (2006).

[6] L. Barrón-Palos et al., Nucl. Phys. A779, 318 (2006).

[7] R. Policroniades, A. Varela, G. Murillo, E. Moreno, E. Chávez, M. E. Ortiz, and A. Huerta, Rev. Mex. Phys. 53, 74 (2007).

[8] E. Chávez, A. Huerta, M. E. Ortiz, P. Rodríguez, F. Favela, D. Marín, E. Moreno, G. Murillo, R. Policroniades, and A. Varela., Rev. Mex. Phys. 53-S6, 13 (2007).

[9] A. Huerta, R. Guerrero, Q. Curiel, J. Huelgas S, P. Rodríguez, F. Favela, D. Marín, M. Ortiz, L. Barrón-Palos, E. Chávez, E. Moreno, G. Murillo, R. Policroniades, and A. Varela, Rev. Mex. Phys. 54, 9 (2008).

[10] E. Chávez, L. Barrón-Palos, Q. Curiel, R. Guerrero, A. Huerta, M. E. Ortiz, E. Moreno, G. Murillo, R. Policroniades, and A. Varela, AIP Conf. Proc. 1099, 84 (2009). 
[11] H. Schmidt, H. Cederquist, R. Schuch, L. Bagge, A. Kllberg, J. Hilke, K.-G. Rensfelt, V. Mergel, M. Achler, R. Drner, L. Spielberger, O. Jagutzki, H. Schmidt-Bcking, J. Ullrich, H. Reich, M. Unverzagt, W. Schmitt, and R. Moshammer, Hyperfine Interact. 108, 339 (1997).

[12] K. Zapfe, B. Braun, H. Gaul, M. Grieser, B. Povh, M. Rall, E. Steffens, F. Stock, J. Tonhuser, C. Montag, F. Rathmann, D. Fick, and W. Haeberli, Rev. Sci. Instrum. 66, 28 (1995).

[13] A. Gruber, W. Bourgeois, B. Franzke, A. Kritzer, and C. Treffert, Nucl. Instrum. Methods Phys. Res., Sect. A 282, 87 (1989).

[14] T. Hosokai, K. Kinoshita, T. Watanabe, K. Yoshii, T. Ueda, A. Zhidokov, M. Uesaka, K. Nakajima, M. Kando, and H. Kotaki, in Proceedings of the 8th European Particle Accelerator Conference, Paris, 2002 (EPS-IGA and CERN, Geneva, 2002), pp. 981-983.

[15] D. Shapira, J. L. C. Ford, R. Novotny, B. Shivakumar, R. L. Parks, and S. T. Thornton, Nucl. Instrum. Methods Phys. Res., Sect. A 228, 259 (1985).

[16] D. Shapira, J. G. Del Campo, J. L. C. Ford, Jr, B. Shivakumar, P. H. Stelson, B. A. Harmon, R. L. Parks, and S. T. Thornton, Nucl. Instrum. Methods Phys. Res., Sect. B 10-11, 436 (1985).

[17] A. Lemut et al., Phys. Lett. B 634, 483 (2006).

[18] A. Kontos, D. Schürmann, C. Akers, M. Couder, J. Görres, D. Robertson, E. Stech, R. Talwar, and M. Wiescher, Nucl. Instrum. Methods Phys. Res., Sect. A 664, 272 (2012).

[19] J. Ullrich, R. Moshammer, A. Dorn, R. Drner, L. P. H. Schmidt, and H. Schmidt-Bcking, Rep. Prog. Phys. 66, 1463 (2003).

[20] V. Tzoganis, A. Jeff, and C. P. Welsch, Vacuum 109, 417 (2014).

[21] V. Tzoganis and C. P. Welsch, Appl. Phys. Lett. 104, 204104 (2014).

[22] Y. Hashimoto, T. Fujisawa, T. Morimoto, Y. Fujita, T. Honma, S. Muto, K. Noda, Y. Sato, and S. Yamada, Nucl. Instrum. Methods Phys. Res., Sect. A 527, 289 (2004).

[23] T. Fujisawa, Y. Hashimoto, T. Morimoto, and Y. Fujita, Nucl. Instrum. Methods Phys. Res., Sect. A 506, 50 (2003).
[24] E. Takada, in Proceeding of the 10th International Conference on Nucleus-Nucleus Collisions (NN2009) [Nucl. Phys. A834, 730c (2010)].

[25] S. Pellegrino, L. Beck, and P. Trouslard, in Proceedings of the Sixteenth International Conference on Ion Beam Analysis [Nucl. Instrum. Methods Phys. Res., Sect. B 219-220, 140 (2004)].

[26] A. Gurbich and S. Molodtsov, in Proceedings of the Eighteenth International Conference on Ion Beam Analysis [Nucl. Instrum. Methods Phys. Res., Sect. B 266, 1206 (2008)].

[27] D. Scott, P. Portner, J. Nelson, A. Shotter, A. Mitchell, N. Chant, D. Montague, and K. Ramavataram, Nucl. Phys. A141, 497 (1970).

[28] A. Valek, T. Vertse, B. Schlenk, and I. Hunyadi, Nucl. Phys. A270, 200 (1976).

[29] D. Shapira and F. Favela (private communication).

[30] J. A. D. Ackroyd, Fluid Mechanics Considerations in Supersonic Jet Target Design (Science Research Council, Daresbury Laboratory, England, 1975).

[31] P. Pritchard, Fox and McDonald's Introduction to Fluid Mechanics (John Wiley \& Sons, New York, 2011).

[32] H. Liepmann and A. Roshko, Elements of Gasdynamics, Dover Books on Aeronautical Engineering Series (Dover Publications, New York, 1957).

[33] M. Mayer, AIP Conf. Proc. 475, 541 (1999).

[34] A. Gurbich, Ion beam nuclear data library (IBANDL), 2014, web application for cross sections.

[35] NASA, Earth fact sheet, 2013, typical air abundance variables.

[36] F. Favela, E. Chávez, O. de Lucio, E. Andrade, and M. E. Ortíz, J. Phys. Conf. Ser. 578, 012001 (2015).

[37] H. Becker, L. Buchmann, J. Görres, K. Kettner, H. Kräwinkel, C. Rolfs, P. Schmalbrock, H. Trautvetter, and A. Vlieks, Nucl. Instrum. Methods Phys. Res. 198, 277 (1982).

[38] K. Chipps, U. Greife, D. Bardayan, J. Blackmon, A. Kontos, L. Linhardt, M. Matos, S. Pain, S. Pittman, A. Sachs, H. Schatz, K. Schmitt, M. Smith, and P. Thompson, Nucl. Instrum. Methods Phys. Res., Sect. A 763, 553 (2014).

[39] R. Brun, F. Rademakers et al., ROOT web page, http://root .cern.ch/. 